\title{
NOTA
}

\section{Negatividad: La figura de Sócrates en la obra de Kierkegaard ${ }^{l}$}

\author{
Negativity: The figure of Socrates in \\ Kierkegaard's work
}

\author{
LAURA LLEVADOT \\ Universidad de Barcelona
}

Recibido: 27-10-2008 Aprobado definitivamente: 12-11-2008

\section{RESUMEN}

El objetivo de este artículo es explorar la relación entre el pensamiento de Kierkegaard y la figura de Sócrates con el fin de comprender por qué Kierkegaard concibe su tarea como la de un «Sócrates cristiano». Mi argumentación seguirá los siguientes puntos: En primer lugar analizaré la noción kierkegaardiana del «pensador existente» en la figura de Sócrates. En segundo lugar, mostraré la oposición entre la negatividad de la ironía socrática y la positividad de Platón y la sofística, tal y como Kierkegaard la analiza en El concepto de ironía. Finalmente trataré de demostrar la diferencia entre el humor kierkegaardiano y la ironía socrática, ambos igualmente negativos.

PALABRAS CLAVE

PENSADOR EXISTENTE, SÓCRATES, IRONÍA, NEGATIVIDAD, HUMOR

\section{ABSTRACT}

The aim of this article is to explore the relationship between Kierkegaard's thought and Socrates in order to understand why Kierkegaard calls himself a «Christian Socrates». My paper will develop the following points: Firstly I will analyze Kierkegaard's notion of the «Existent Thinker» in the model of Socrates. Secondly, I will show the difference between the negativity of Socratic irony and the positivity of Platonic and Sophistic position, which Kierkegaard analyzes in On the concept of Irony. Finally, I will try to show de difference between Kierkegaardian humour and Socratic irony, both equally negative modes of thought.

1 Una primera versión de este texto fue leída en el I Congreso Internacional de Filosofía Griega que tuvo lugar en la Universidad de las Islas Baleares, Mallorca, en Abril de 2008.

(C) Contrastes. Revista Internacional de Filosofía, vol. XIV (2009), pp. 269-280. ISSN: 1136-4076

Licenciatura de Filosofía, Universidad de Málaga, Facultad de Filosofía y Letras

Campus de Teatinos, E-29071 Málaga (España) 


\section{KEY WORDS \\ EXISTENT THINKER, SOCRATES, IRONY, NEGATIVITY, HUMOUR}

«SE OPINA QUE EL MUNDO NECESITA UNA REPÚBLICA, un nuevo orden social, e incluso una nueva religión: pero nadie piensa que de lo que más necesidad tiene el mundo, en virtud de la confusión en la que le ha dejado tanto saber, es de otro Sócrates», así se pronuncia Kierkegaard en la Enfermedad mortal (EM, 177/SV3, XV, 142). ${ }^{2}$ Podría decirse que Sócrates es para Kierkegaard una suerte de intercesor -por utilizar la terminología de Deleuze-, un personaje conceptual y una determinación existencial que permite a Kierkegaard crear su propio pensamiento. De hecho, a menudo Kierkegaard se presenta a sí mismo como un «Sócrates cristiano» y se vanagloria de haber introducido la ironía y la mayéutica, a través de sus pseudónimos, en el pensamiento moderno. ¿Qué conexión existe entre el pensamiento existencial kierkegaardiano y la ironía socrática? ¿Qué ve Kierkegaard en Sócrates que merezca ser introducido en la modernidad precisamente para corregir su tendencia? ¿Por qué Sócrates fue y sigue siendo el correctivo que la época necesita? Lo que, según Kierkegaard, diferencia a Sócrates de «una república, un nuevo orden social, o una nueva religión» es su negatividad. Frente a la positividad del saber, de las ideologías políticas o religiosas, Sócrates encarna la negatividad necesaria para liberar al pensamiento de la sumisión a los dictámenes de la actualidad. En este sentido Sócrates representa el tipo de pensador que Kierkegaard mismo quería ser, un pensador privado, un pensador existente, siempre heterogéneo respecto a las exigencias de la ciudad. Kierkegaard retomará de Sócrates esta negatividad, tanto en su modo de pensar la existencia como en su estrategia conceptual. Ciertamente, hay un punto en que sus caminos se bifurcan, allí donde Kierkegaard hace valer el humor frente a la ironía, allí donde la dialéctica cualitativa sustituye a la dialéctica negativa de corte socrático. Sin embargo, aún en ese recorrido propiamente kierkegaardiano, la negatividad socrática hace notar su presencia.

2 En lo que se refiere a la obra de Kierkegaard señalamos, en primer lugar a la traducción española [Obras y Papeles de Kierkegaard. Ed. Guadarrama, Madrid, 1965, trad. a cargo de Demetrio Gutiérrez Rivero y para El concepto de ironía, la nueva traducción española: Escritos Søren Kierkegaard, vol. 1, editado por Rafael Larrañeta, Darío González y Begonya Sàez Tajafuerce. Madrid: Editorial Trotta, 2000] si la hay o en su defecto a la traducción francesa [S $\phi r e n$ Kierkegaard. Oeuvres Complètes. Paris: Éditions de L'Orante, 1997], como es el caso del PostScriptum definitivo y no científico a las Migajas Filosóficas (en adelante P.S.). Seguidamente, damos la referencia de la nueva edición danesa de las obras de Kierkegaard, Søren Kierkegaard Skrifter, editado por Niels Jørgen Cappelørn, Joakim Garff, et al., Copenhagen: Gads Forlag, 1999-2007, (en delante SKS), o en su defecto, como es el caso de La enfermedad mortal, S $\phi r e n$ Kierkegaard Samlede Varker (20 vol.), editada por A. B. Drachmann, J. L. Heiberg, y H.O. Lange. Copenhagen: Gyldendal, 1962-1964 (SV3). 
En este sentido este artículo desarrollará las siguientes cuestiones: En primer lugar trataré de mostrar la necesidad que Kierkegaard tiene de introducir el correctivo socrático en el pensamiento moderno. En segundo lugar, analizaré la oposición entre la posición socrática y las posiciones sofística y platónica que Kierkegaard traza en El concepto de ironía en constante referencia a Sócrates. Finalmente compararé la estrategia socrática (como dialéctica negativa) con el pensamiento kierkegaardiano de la dialéctica cualitativa, para medir la distancia pero también la inspiración socrática del pensamiento existencial.

\section{IDEALIDAD Y REALIDAD: EL «GESTO» SOCRÁTICO}

Cuando Kierkegaard reclama en La enfermedad mortal un Sócrates para la época lo hace a su vez ofreciendo un diagnóstico de la misma: el problema de la época es el exceso de saber y la confusión generada por él. Kierkegaard define el saber como la reducción de la realidad operada por la idealidad. Saber es aprehender idealmente, abstractamente, aquello que no se deja pensar, es el movimiento que se opera $a b$ esse ad posse, de la realidad a la posibilidad. Sabemos algo cuando creemos haber captado su esencia, pero la esencia, precisamente, es una determinación del pensamiento que no se confunde con la existencia fáctica. «Todo saber sobre la realidad [Virkelighed] es posibilidad [Mulighed]» (PS, II, 15/ SKS, 288). Kierkegaard sigue aquí las enseñanzas de su maestro Møller, quien definía la ontología -al igual que las matemáticas- como un saber a priori, previo a la experiencia y por lo tanto siempre exterior a la realidad. ${ }^{3}$ La ontología, el saber filosófico por excelencia, es únicamente un saber hipotético, o como diría el último Schelling, argumentando su crítica a la filosofía hegeliana, ${ }^{4}$ un saber meramente «posible», dado que el concepto se

3 P. M. Møller, «Tanker over Muligheden af Beviser for Menneskets Udødelighed, med Hensyn til den nyste derhen hørende Literatur» [Reflexiones sobre la posibilidad de probar la inmortalidad del hombre en referencia a la más reciente literatura sobre el tema], en Maanedsskrift for Litteratur, vol. 17, 1837, pp.1-72 y 422-453 (Existe traducción al francés en H-B. Vergote, Lectures Philosophiques de Søren Kierkegaard: Kierkegaard chez ses contemporaines danois. Paris : Presses Universitaires de France, 1993, pp. 149-213). Sobre la relación entre Kierkegaard y Møller, ver G. Malantschuk, «Søren Kierkegaard og Poul Møller» en Kierkegaardiana, $\mathrm{n}^{\circ} 3$, (1959), editado por Søren Kierkegaard Selskabet y N. Thulstrup, p. 9 (pp. 7-20); y P. Lübcke, «Det ontologiske program hos Poul Møller og Søren Kirkegaard» en Filosofiske Studier, IV, (1983).

4 F. W. J., Schelling, Philosophie de la Révélation (Livre I), trad. fr. de la RCP Schellingiana (CIURS) bajo la dirección de Jean-François Marquet y Jean-François Courtine. Paris : Presses Universitaires de France, 1989, pp. 91-93. [Título Original: Philosophie der Offenbarung, 1841-1842]. 
establece a priori, a espaldas del ser efectivo. El problema de la época es, según Kierkegaard, el exceso de saber, esto es la confusión entre idealidad y realidad, la creencia infundada de que el pensamiento abstracto puede alcanzar lo real. El proyecto kierkegaardiano de pensar la existencia se sitúa, de este modo, en el polo opuesto del saber. No se trata de pensar la cosa a partir de la idea, sino al contrario, de pensar la existencia, esto es la diferencia, entre la realidad y la idealidad, entre el ser y el pensar, entre el existir y su verdad. Entre el ser y el pensar no hay adecuación posible porque la existencia es justo aquello que los separa, de ahí que Kierkegaard defina la existencia como un inter-esse, un entre-ser (PS, II, 14/ SKS, 286). El problema del saber es que al presuponer la adecuación entre realidad e idealidad, entre pensamiento y ser, olvida aquello que los separa, olvida la propia existencia de quien así piensa, de modo que se produce aquí una escisión. El filósofo, el científico, creen pensar la realidad pero mientras lo hacen olvidan su propia realidad, su condición de existentes. Tal vez piensen y sepan muchísimas cosas, tal vez elaboren complejas estructuras conceptuales, pero su existencia no se rige por su pensar sino que transcurre bajo categorías cómodas. Tal y como ya Lukács supo ver, el problema de Kierkegaard es el «gesto»: «el punto en que se cruzan realidad y posibilidad». ${ }^{5}$ Lo que Kierkegaard pretende es introducir la idealidad en la realidad, las formas en la vida -si seguimos la terminología de Lukács. Frente al filósofo o el científico que creen pensar la realidad dejando su existencia intacta, lo que Kierkegaard exige al pensador existente es la reduplicación [Fordobling], la capacidad de ser lo que se dice, de existir como se piensa. La existencia es ciertamente lo que no puede ser pensado de modo abstracto, pero es también lo que exige que el pensamiento intervenga para darle consistencia, para que la vida no sea un mero pasar y el pensamiento un juego abstracto. Introducir el pensamiento en la vida es dejar que éste determine nuestro modo de existencia, que el sujeto que piensa se vea afectado radicalmente, por su pensamiento, en su modo de vivir. De ahí que Lukács afirme que: «el gesto es una necesidad vital de fuerza primigenia; tal vez porque el hombre que quiere ser honesto ha de conseguir de la vida inequivocidad». 6

Es aquí donde la figura de Sócrates interviene como un modelo a seguir en el pensamiento kierkegaardiano. Sócrates fue un pensador honesto, vivió su vida reduplicando su pensar. De la filosofía moderna se puede decir que «La metafísica es abstracción, y nadie existe metafísicamente» (S, 438/SKS, 439), que es imposible llevar a cabo una existencia de filósofo. Sin embargo Sócrates

5 G. Lukács, «La forma se rompe al chocar con la vida», en El alma y las formas, Obras Completas, vol. I. Barcelona: Ed. Grijalbo, 1970, pp. 57-76 [Título original: Die Seele und die Formen, Essays, 1911], p. 58.

6 G. Lukács, op. cit., p. 58. 
existió como filósofo porque reduplicó su pensamiento en su existencia y en este sentido consiguió que su vida fuese inequívoca. Sócrates fue en sentido propio un «pensador existente» pero precisamente porque siempre supo resistirse al saber, a la confusión que implica la creencia en la adecuación entre pensamiento y ser. Es por su oposición al saber que Sócrates puede ser presentado por Kierkegaard como un correctivo existencial frente a la abstracción de la época.

\section{LA NEGATIVIDAD SOCRÁTICA COMO DETERMINACIÓN EXISTENCIAL}

«Ser lo que se dice», «vivir en lo que se comprende», es la tarea de reduplicación que Kierkegaard exige al pensador existente. Su lectura de Sócrates en El concepto de ironía difiere de la de Hegel justo porque Kierkegaard entiende que la ironía, lejos de ser únicamente el momento negativo de un método que es corregido por el momento positivo de la mayéutica, ${ }^{7}$ es para Sócrates una posición existencial que determina la personalidad misma de Sócrates y todo su quehacer. La ironía se caracteriza por la conciencia de la discrepancia existente entre la idea y la cosa, por ver la contradicción que hay entre todo fenómeno real y la idea que de él se hace el hombre. En este sentido la ironía supone una ruptura respecto a la conciencia inmediata del helenismo: «En la venturosa Grecia, la esencia estaba unida al fenómeno en tanto que determinación inmediata de la naturaleza» (CI, 246/ SKS, 257). La conciencia inmediata, representada en este caso por el helenismo, no ve contradicción alguna entre esencia y apariencia, entre idea y fenómeno, de ahí que sea relativamente fácil fundar una eticidad garantizada por la existencia real de los dioses helénicos, establecer valores que se siguen necesariamente del orden real de las cosas. Pero si la ironía representa una ruptura respecto a esta conciencia inmediata del helenismo es porque, antes que ella, ha aparecido una ruptura anterior que no se da por tal y que es la sofística. La sofística representa para Kierkegaard el despertar de la reflexión, lo que significa poner en duda la realidad fenoménica, y aún más la realidad de aquello que aparece determinante para los hombres: las leyes, las normas, la eticidad común y general (CI, 240/SKS, 249). En este sentido la sofística aporta un correctivo negativo a lo comúnmente aceptado como verdadero. Su verdad negativa consiste en la capacidad para mostrar la realidad como hipotética. Pero por otro lado, la sofística no se conforma con suspender toda realidad sino que acto seguido aplica categorías arbitrarias para otorgar uno u otro sentido a la

7 La lectura de Sócrates como «Jano bifronte», con un momento negativo y otro positivo, la ofrece Hegel en sus Lecciones sobre la historia de la filosofía, II. México: Fondo de Cultura Económica, 2005. Para una comparación detallada de la lectura de Kierkegaard y Hegel acerca de Sócrates, ver: S. Kofman, Socrate(s), Paris: Galilée, 1989. 
realidad antes negada. Es de este modo que la sofística se traiciona a sí misma en cuanto su primera reflexión es contrarrestada por el mero «arte de hablar», de aplicar las categorías correctas para que todo tome un sentido dirigido y predeterminado al acto mismo de la reflexión negativa. Así debe comprenderse la afirmación de Kierkegaard según la cual:

Comparada con la conciencia inmediata, que de manera totalmente inocente acepta con infantil ingenuidad lo que se da, esta cultura es, por tanto, negativa, y es demasiado astuta como para ser inocente; comparada con el pensamiento, en cambio, es positiva en alto grado. En la primera de sus formas, esta cultura hace que todo se tambalee; en la segunda, en cambio, da a cualquier discípulo honesto la capacidad de sujetar las cosas. (CI, 240/ SKS, 250).

La sofística es así comprendida como un «saber que se independiza de la eticidad» (CI, 238/ SKS, 247), es decir, un saber que en cuanto negativo rompe con la ingenuidad helénica así como con sus usos y costumbres, pero a la vez reinventa cualquier verdad en función de los intereses en juego con el fin de volver a sujetar la realidad e imponer sus nuevas leyes y normas.

Lo que en este fragmento es llamado «pensamiento» y que Kierkegaard distingue de la sofística justo por su carácter negativo es la ironía que Sócrates encarna. Pero la ironía se distingue a su vez de otro intento de reordenar la realidad una vez ésta ha sido puesta en tela de juicio por la sofística. Nos referimos a la tentativa platónica a la que Kierkegaard hace alusión en el siguiente fragmento:

Cuando se ha hecho que todo se tambalee, ¿qué podrá ser lo firme que salve la situación? O bien es lo universal (lo bueno, etc.), o bien el sujeto finito, su arbitrariedad, sus inclinaciones, etc. Los sofistas tomaron este último camino. (CI, 241/ SKS, 250)

Sócrates y Platón personifican la reacción contra esta recreación interesada de la realidad que los sofistas llevan a cabo con la habilidad de las categorías. La diferencia entre ambos es que la posición platónica es positiva, busca el bien, lo universal -aunque el método para hallarlo sea negativo-, mientras que la posición socrática es absolutamente negativa. Kierkegaard va estableciendo las diferencias entre ambas posiciones a lo largo de la obra, pero están recogidas especialmente al final de la primera parte: «La posición de Sócrates concebida como ironía», cuando se cierra el análisis de la obra de Platón. Allí se nos dice:

[...] [H]ay dos tipos de ironía y dos tipos de dialéctica. Hay una ironía que es un mero stimulus para el pensamiento, que lo estimula cuando se pone perezoso, 
que lo corrige cuando se descarría. Hay una ironía que opera por sí misma y que es ella misma el terminus hacia el que se tiende. Hay una dialéctica que, en constante movimiento, no cesa de velar por impedir que la interrogación se deje engatusar por una percepción fortuita, que siempre e incansablemente se apresta a sacar el problema a flote cuando afonda, que, en suma, siempre se las arregla para mantener el problema en suspenso, y que por eso y en función de eso busca resolverlo. Hay una dialéctica que, partiendo de la idea abstracta, quiere hacer que ésta se despliegue en determinaciones más concretas, una dialéctica que quiere construir la realidad con la idea. (CI, 172/ SKS, 172-173).

La tesis que aquí Kierkegaard defiende es que en los mismos diálogos platónicos se puede percibir la lucha entre ambas posiciones, la socrática y la platónica. A la posición platónica le corresponde un tipo de ironía que es únicamente método para despertar el pensamiento y hacerlo llegar a la plenitud de la idea. Esto lo explica Kierkegaard en otro lugar cuando afirma que el preguntar platónico es un preguntar especulativo puesto que presupone la plenitud de una respuesta posible. No se pregunta con el mero fin de polemizar irónicamente con el adversario, sino con el fin de hallar una respuesta verdadera y por lo tanto presuponiendo que ésta existe. A esta ironía le incumbe una dialéctica que pretende «construir la realidad con la idea», reorganizar la realidad una vez ésta ha sido mostrada como hipotética y tras haber sido hallada la idea que le corresponde para dotarla de sentido y de verdad.

Por el contrario la posición socrática, en cuanto irónica, es mucho más corrosiva y escéptica que la platónica. En Sócrates la ironía se convierte en un fin en sí misma, no en un método para hallar la verdad. El preguntar irónico no presupone la plenitud de la respuesta posible sino que da por supuesto el vacío y no trata sino de mostrarlo, de hacerlo valer frente a toda respuesta que quisiera saturar la brecha abierta por el pensamiento. Del mismo modo, la dialéctica socrática no pretende construir la realidad, sino bien al contrario lo que busca es mantener el problema en suspenso, en tanto problema. Es por ello que Kierkegaard habla a propósito de Sócrates de una dialéctica negativa (CI, 196/ SKS, 200), una dialéctica que partiendo de la realidad para llegar a la idea no llega sin embargo nunca a ésta, sino que vuelve a retrotraerse a la realidad sin encontrar determinación alguna que la sujete. ${ }^{8}$

Así pues, Sócrates encarna la posición absolutamente negativa de la ironía en virtud de la cual es heterogéneo respecto a la realidad circundante. Lo único que se salva aquí es la propia subjetividad de quien ejerce la ironía y

8 En otro pasaje Kierkegaard afirma que si bien Sócrates alcanzó la idea de dialéctica no llegó jamás a concebir la dialéctica de la idea, mostrando así la oposición entre la posición irónica y negativa de Sócrates y la posición especulativa de Hegel. (CI,169/SKS,170). 
de la que Sócrates mismo da cuenta en su propio existir cuando da a conocer su indiferencia hacia la muerte, o su talante experimental cuando se muestra como sujeto moralmente libre, cuando exhibe su ataraxia irónica que le permite jugar libremente con las ideas morales sin hipostasiar ninguna, a diferencia de la positividad de los sofistas. La peligrosidad de Sócrates para la ciudad que Kierkegaard trata de recuperar en la modernidad consiste en esta negatividad irónica: «Si Sócrates hubiese tenido una positividad que imponer, la consecuencia habría sido que él y los sofistas se pusiesen a hablar al mismo tiempo, pues el saber sofístico era tan tolerante como la religión de los romanos, no tenían nada en contra de que hubiese un sofista más, una tienda más» (CI, 244/ SKS, 254). Aquí Kierkegaard recupera la enseñanza socrática: no plantar tiendas, ni sofísticas ni especulativas, no ofrecer saber ni positividad alguna, dejar de este modo de producir confusión, dejar de darle a la época lo que pide: «pues hay -dice Kierkegaard- fundamentalmente en el Estado griego y en cierta medida en todo Estado, una ignorancia que debe ser considerada un crimen» (CI, 211/ SKS, 218). Es en este lugar del crimen donde Kierkegaard, como Sócrates, sitúa la tarea del pensar.

\section{IRONÍA Y HUMOR: DE LA DIALÉCTICA NEGATIVA A LA DIALÉCTICA CUALITATIVA}

El pensamiento existencial que Kierkegaard propone es así un pensamiento negativo que exige del pensador la reduplicación, una determinación de existencia. Ahora bien, hay un punto donde Kierkegaard abandona a Sócrates para iniciar su propia andadura, es el punto en que Kierkegaard hace valer lo cristiano sobre toda especulación. Pero es justo aquí donde es necesario tener en cuenta que lo cristiano no es presentado como una positividad más, como una doctrina o una «tienda», sino como un pensamiento existencial que, como la ironía socrática, tiene en cuenta la inadecuación del pensamiento y la realidad, de ahí el recurso al humor. La diferencia entre la ironía socrática y el humor cristiano es la «conciencia de la falta» (PS, II, 235/SKS, 7, 503), lo que la dogmática cristiana llamará el pecado, esto es la conciencia de que ni siquiera la subjetividad es libre, sino que la subjetividad es la no-verdad:

Pero porque el humor oculta siempre un dolor escondido, comporta así mismo una simpatía de la que la ironía está desprovista puesto que lo que ésta busca es hacerse valer; de modo que su simpatía no es sino indirecta, no se aplica sobre persona alguna, sino a la idea de hacerse valer uno mismo. Es por ello que en la mujer encontramos a menudo el humor pero jamás la ironía. Una naturaleza verdaderamente femenina verá siempre en la ironía una suerte de crueldad. (PS, II, 235/SKS, 7, 502) 
Lo que distingue la ironía del humor es pues el dolor que implica saber que ni siquiera la subjetividad está a salvo. Es este dolor el que produce la simpatía, la conexión entre aquellos cuya existencia se sabe indefinidamente alejada de su verdad y que por eso mismo no se limitan a relativizar la realidad haciendo que parezca hipotética sino que la buscan, la desean, a sabiendas de no poder hallarla por sus propios medios. Es este deseo paradójico de querer creer contra toda racionalidad, de creer en virtud del absurdo, lo que caracteriza la condición trágica del cristianismo que Kierkegaard renueva. Desde esta posición vemos aparecer un cambio de estrategia frente a la positividad del saber y la especulación. Que la realidad no coincide con la idea puede mostrarse a través de la estrategia negativa que Sócrates encarna. Se trata entonces de no afirmar nada, de llevar a cabo un preguntar negativo cuya única función es mostrar el fracaso necesario de toda respuesta posible. Pero se puede también, y ésta es la estrategia propiamente kierkegaardiana, afirmarlo todo y a la vez, multiplicar las respuestas positivas y hacer que sea el lector, cuál discípulo socrático, el que interiorice la pregunta y genere la respuesta que ha de modificar su modo de existir. Esto es lo que Kierkegaard llama «dialéctica cualitativa», frente a la dialéctica negativa de corte socrático. La dialéctica cualitativa es un «arte de las distinciones» [en forskelliggørende Kunst], se trata de mostrar bajo la univocidad y universalidad del concepto las diferencias reales que éste esconde. Así por ejemplo, la misma figura de Sócrates aparece en la obra de Kierkegaard encarnando diversas posiciones existenciales, no solamente la ironía -tal y como se presenta en El concepto de ironía-, sino que en las Migajas Filosóficas Sócrates es identificado voluntariamente con la posición platónica, mientras que en el Post-Scriptum Sócrates va a ocupar la posición de lo religioso. ${ }^{9}$ Cada una de las obras ofrece una distinción entre posiciones opuestas sin mediación posible (lo socrático/lo platónico en El concepto de ironía; lo socrático-platónico/lo cristiano en Migajas Filosósficas; lo socrático-

9 No es éste el lugar para analizar los desplazamientos que la figura de Sócrates sufre en la totalidad de la obra de Kierkegaard, valga sin embargo señalar los lugares en que Kierkegaard hace relevantes dichos desplazamientos, así por ejemplo cuando afirma en el Post-Scrpitum: «Es el momento de aclarar un punto delicado en el plan de las Migajas; [...] Atribuyendo a Sócrates la tesis de que todo conocimiento es una reminiscencia, se hace de él un filósofo especulativo, mientras que él era un pensador existente para quien lo esencial era existir. [...] Acentuar la existencia, lo cual implica interioridad, es lo propio de Sócrates; pero lo propio de Platón es engarzarse en la persecución de la reminiscencia y de la inmanencia» (PS., I, 192/ SKS, 7, 189). Y al poco afirma: «Pero justamente porque supera la especulación, Sócrates, correctamente presentado, ofrece una cierta analogía y parecido con el ensayo y la experiencia que Migajas presenta como superando realmente el punto de vista socrático» (PS, I, 192 / SKS, 7, 189). He trabajado esta cuestión en el artículo «Repetición y representación: Sobre la filosofía segunda de Søren Kierkegaard», Kierkegaardiana, n. 23, (2004) Copenhaguen, pp. 162-175. 
religioso/lo religioso cristiano en el Post-Scriptum), pero a la vez las nuevas distinciones que se establecen no invalidan las precedentes sino que son internas a las mismas. Como afirma Brézis, la diferencia en esta filosofía no es nunca representativa, no se trata de diferencias entre identidades opuestas, sino de diferencias en el seno de una misma identidad. ${ }^{10}$ Es el mismo Sócrates el que, al ser repetido, va desplazándose de sí mismo, es la misma determinación la que va cambiando de posición a medida que se interioriza, que profundiza en su realidad. La filosofía existencial hace la diferencia interna a la conceptualización. ${ }^{11}$ No se trata de oponer conceptos y establecer su distancia respectiva, ni siquiera se trata de concatenar conceptos de manera deductiva para llegar a una determinada verdad, se trata por el contrario de devolver al concepto lo que éste quita a la realidad, a la realidad subjetiva, es decir su complejidad, su diferenciación interna, aquello que escapa a su determinación abstracta. De este modo la determinación de lo socrático sirve como ejemplo para ver el modo de operar de esta dialéctica, el modo de diferenciar y distinguir en el seno mismo de una determinación de la subjetividad. Las diferentes posicio-

10 Así afirma Brézis en relación con la diferencia que supone la excepción respecto a lo general en la filosofía de Kierkegaard: «La différence qui caractérise l'exception n'est jamais chez Kierkegaard extérieurement assignable. Elle ne se constitue jamais dans un rapport d'extériorité à ce dont elle diffère. Elle est d'autant plus insaisissable qu'elle est différence à l'intérieur d'une identité. [...] Sa différence ne se laissant pas déterminer de manière univoque (comme intérieure ou extérieur au genre), elle est essentiellement bâtarde. Elle l'est du moins pour les contemporains car, après coup, son équivoque vient à disparaître, occulté dans une représentation qui la ressaisit comme une différence purement extérieur.» (D. Brézis, Temps et Présence. Essai sur la conceptualité kierkegaardienne, Paris : Vrin, 1991, p. 234).

11 Algunos intérpretes han insistido en la cuestión del desplazamiento en la obra de Kierkegaard. Es especialmente en el ámbito de la crítica literaria que esta cuestión ha tomado su máxima relevancia. La producción de la «diferencia» en virtud de la repetición ha sido acometida, al menos, en dos sentidos: 1) En primer lugar, como análisis de la diferencia que Kierkegaard opera respecto a los modelos literarios originarios que retoma modificándolos. En este sentido, destaca la obra de L. Mackey, Kierkegaard: a Kind of Poet. Philadelphia: University of Pennsylvania Press, 1971. 2). En segundo lugar, se ha estudiado también, la diferencia o «distancia crítica» que Kierkegaard establece al utilizar conceptos recibidos de la tradición, en especial de Hegel, para vaciarlos de su contenido originario y atribuirles una nueva función (lo que Poole llama, tomando en préstamo el término, palaeonymic displacement). Pero también se ha hablado de la diferencia interna de la conceptualidad kierkegaardiana, en el sentido que muchos de sus conceptos no son unívocos sino que toman significados diversos en cada obra. Sobre estos dos últimos aspectos, cabe destacar la interpretación de Roger Poole quien a propósito del concepto de «sí mismo» afirma: «I would like to emphasise that all these versions of the self are works of difference in the sense that Kierkegaard is differentiating between concepts in his own corpus. His art is, as he describes it, en forskelliggфrende Kunst, an art of making distinctions.» p. 441, en R. Poole, «Towards a Theory of Responsible Reading: How to Read and Why», en Kierkegaard Studies. Yearbook (2002), pp. 395-442. 
nes que la determinación de lo socrático encarna en estas tres obras no son señaladas de modo lineal, no están dichas, sino que son hechas en virtud del movimiento propio de la dialéctica. Cada desplazamiento viene precedido por un salto, cada obra pone en marcha la maquinaria de la diferenciación sin que haya una continuidad deductiva entre ellas.

Ahora bien, el humor que supone multiplicar las positividades, descubrir bajo la univocidad positiva del concepto una multiplicidad de respuestas posibles, precisamente porque no hay nada que salvar, ni siquiera la subjetividad de quien piensa, no está exento de ironía. De hecho, el humor sirve a la negatividad irónica de un modo más radical que la propia ironía:

Una réplica irónica no puede hacer irónica una situación; puede a lo sumo hacer ver que la situación lo es; por el contrario una réplica humorística puede hacer irónica una situación. El ironista busca hacerse valer e impide que la situación se forme, pero el dolor secreto del humorista comporta una simpatía la cual contribuye a crear la situación [Situationen] irónica. (PS, II, 234/ SKS, 7, 501)

La estrategia absolutamente negativa de la ironía tiene algo positivo, salvar al sujeto irónico de su comercio con la realidad. Lo que aquí Kierkegaard llama situación es la realidad que el ironista cancela. Por el contrario, lo humorístico que consiste en ofrecer una multiplicidad de respuestas positivas a la vez, de modo que se revocan las unas a las otras, hace irónica la situación, esto es, muestra que la realidad no coincide nunca con la idea pero exige en el mismo gesto la «idealidad de la realidad», que el lector, ante las posibilidades existenciales que se le ofrecen, se decida por la inequivocidad en su existir. Esto es lo que hace Kierkegaard con sus obras pseudónimas, dar a ver posibilidades existenciales contradictorias a la vez sin que ninguna de ellas sea el modelo a seguir. Es en este sentido que su dialéctica cualitativa es más negativa que la estrategia irónica. Produce en el lector no ya el fracaso sino el silencio necesario para que reestablezca una relación no abstracta con su propia realidad existencial.

Sería pues un error creer que la obra de Kierkegaard nos propone una filosofía positiva o una doctrina a seguir: sea el existencialismo o el cristianismo. Si Kierkegaard aprendió algo de Sócrates fue a no plantar tiendas o, en una radicalización de la posición socrática, a plantar tantas que fuese imposible decidirse por una de ellas. El humor kierkegaardiano tiene por finalidad el silencio y su reduplicación en la existencia, algo que como la ignorancia, cualquier estado debe considerar un crimen. 
Laura Llevadot es Profesora Lectora de Filosofía Contemporánea en el Departamento de Historia de la Filosofía, Estética y Filosofía de la Cultura, Facultad de Filosofía, Universidad de Barcelona.

Publicaciones recientes:

«El individuo singular: El cine de Lars von Trier a la luz de Kierkegaard», Themata. Revista de Filosofía, num. 39, Universidad de Sevilla, 2007, pp. 435-441.

«El estatuto de la ficción en Nietzsche y Foucault», Convivium. Revista de Filosofía, n. 21, Universitat de Barcelona, 2008, pp. 71-82.

Dirección postal:

C/ Comte d'Urgell, 239, 6-3, 08036 Barcelona.

Dirección electrónica: laullevadot@ub.edu 